Article

\title{
A Symmetric FBF Method for Solving Monotone Inclusions
}

\author{
Aviv Gibali ${ }^{1,2, *,+(1)}$ and Yekini Shehu ${ }^{3,+}+(\mathbb{C}$ \\ 1 Department of Mathematics, ORT Braude College, Karmiel 2161002, Israel \\ 2 The Center for Mathematics and Scientific Computation, University of Haifa, Mt. Carmel, Haifa 3498838, Israel \\ 3 Department of Mathematics, Zhejiang Normal University, Jinhua 321004, China; yekini.shehu@unn.edu.ng \\ * Correspondence: avivg@braude.ac.il \\ + These authors contributed equally to this work.
}

Received: 17 July 2020; Accepted: 2 September 2020; Published: 4 September 2020

\begin{abstract}
The forward-backward-forward (FBF) splitting method is a popular iterative procedure for finding zeros of the sum of maximal monotone and Lipschitz continuous monotone operators. In this paper, we introduce a forward-backward-forward splitting method with reflection steps (symmetric) in real Hilbert spaces. Weak and strong convergence analyses of the proposed method are established under suitable assumptions. Moreover, a linear convergence rate of an inertial modified forward-backward-forward splitting method is also presented.
\end{abstract}

Keywords: forward-backward-forward splitting; reflected step; weak convergence; linear convergence; Hilbert spaces

\section{Introduction}

In this paper we are concerned with solving monotone inclusion problems and introducing a new self-adaptive, reflected forward-backward-forward method for solving it. Monotone inclusions appear naturally and play an important role in many applied fields, such as fixed point problems, equilibriums, and many more, as, for example, in [1-8]. More precisely, various problems in signal processing, computer vision and machine learning can be modelled mathematically using this formulation, see for example [9] and the references therein.

Let us recall the definition of the monotone inclusion problem. Given a maximal monotone operator $B: H \rightarrow 2^{H}$ and a Lipschitz continuous monotone operator $A: H \rightarrow H$ defined on a real Hilbert space $H$; the monotone inclusion problem is formulated as finding a point $x \in H$ such that

$$
0 \in(A+B) x
$$

One of the simplest and most popular methods for solving (1) is the well-known forward-backward splitting method, introduced by Passty [7] and Lions and Mercier [5]. The iterative step of the method is phrased as follows.

$$
x_{n+1}=J_{\lambda_{n}}^{B}\left(I-\lambda_{n} A\right) x_{n}, \lambda_{n}>0,
$$


where $J_{\lambda_{n}}^{B}:=\left(I+\lambda_{n} B\right)^{-1}$ denotes the resolvent of the maximal monotone operator $B$. Tseng, in [10], introduced a modification of (2) that includes an extra step that enables us to obtain convergence under weaker assumptions than those mentioned above.

The Tseng [10] iterative step is formulated as follows.

$$
\left\{\begin{array}{l}
y_{n}=J_{\lambda_{n}}^{B}\left(x_{n}-\lambda_{n} A x_{n}\right), \\
\left.x_{n+1}=y_{n}+\lambda_{n}\left(A x_{n}-A y_{n}\right)\right), \quad n \geq 1,
\end{array}\right.
$$

where $\lambda_{n} \in\left(0, \frac{1}{L}\right)$ ( $L$ is the Lipschitz constant of $A$ ) is obtained using an Armijo line search rule, as seen in ([10] (2.4)). The forward-backward-forward algorithm (3) has been studied extensively in the literature due to its applicability-e.g., in [11-21].

Recently, Malitsky and Tam [22] introduced the following forward-reflected-backward splitting method for solving (1).

$$
x_{n+1}=J_{\lambda_{n}}^{B}\left(x_{n}-\lambda_{n} A x_{n}-\lambda_{n-1}\left(A x_{n}-A x_{n-1}\right)\right),
$$

where $\lambda_{n} \in\left[\epsilon, \frac{1-2 \epsilon}{2 L}\right], \epsilon>0$ and $\lambda_{n}$ is defined via a line search procedure, as seen in ([22] Algorithm 1).

Csetnek et al., in [23], proposed the following iterative step for solving (1).

$$
\left.x_{n+1}=J_{\lambda}^{B}\left(x_{n}-\lambda A x_{n}\right)-\lambda\left(A x_{n}-A x_{n-1}\right)\right),
$$

where $\lambda \in\left[\epsilon, \frac{1-3 \epsilon}{3 L}\right], \epsilon>0$.

Observe that the iterative methods (4) and (5) coincide when $B=0$ but (4) is more general due to the choice of $\lambda_{n}$. In any case, a major drawback of these two methods is the prior knowledge of the Lipschitz constant of $A$ and, in most applications, this is either unknown or difficult to approximate. Furthermore, when a line search procedure is used as an inner loop, this might include extra computations and hence results in slow convergence and then could make the method inefficient.

Very recently, Hieu et al., in [24], proposed a self-adaptive forward-backward splitting variant that does not depend on the Lipschitz constant of $A$ and no line search procedure is required. Choose $\lambda_{1}>0$ and $\mu \in\left(0, \frac{1}{2}\right)$.

$$
\left\{\begin{array}{l}
x_{n+1}=J_{\lambda_{n}}^{B}\left(x_{n}-\lambda_{n} A x_{n}-\lambda_{n-1}\left(A x_{n}-A x_{n-1}\right)\right), \\
\lambda_{n+1}=\min \left\{\frac{\mu\left\|x_{n+1}-x_{n}\right\|}{\left\|A x_{n+1}-A x_{n}\right\|}, \lambda_{n}\right\} .
\end{array}\right.
$$

Motivated by the above recent developments in the field of algorithms for solving inclusion problems (1), our contributions in this paper are:

1. We propose a new reflected forward-backward-forward iterative method for solving inclusion problems that has a different structure from the methods proposed in [10,22-24].

2. Our scheme is a self-adaptive procedure that does not require prior knowledge of the Lipschitz constant of $A$ and no line search procedure is needed.

3. We also propose a modification of the forward-backward-forward method with inertial extrapolation step and obtain linear convergence result under some standard assumptions.

For deeper understanding and motivation, we next present the relations between dynamical systems and monotone inclusions. 


\section{Dynamical Systems and Monotone Inclusions}

The forward-backward splitting method (2) can be interpreted as a discretization of the dynamical system (see $[25,26])$

$$
\dot{x}(t)+x(t)=J_{\lambda}^{B}(x(t)-\lambda A x(t))
$$

which consequently takes the form

$$
-\dot{x}(t) \in \lambda B(\dot{x}(t)+x(t))+\lambda A x(t)
$$

as a monotone inclusion (1). Therefore, (7) can be considered as the dynamical system for the monotone inclusion (1), where $A$ is co-coercive.

Now, let us consider the monotone inclusion (1) for which $A$ is monotone and Lipschitz continuous on $H$. To solve (1), let us consider the dynamical system, in the spirit of (8):

$$
\dot{x}(t)+\alpha(t) x(t)=\alpha(t)\left[J_{\lambda}^{B}(I-\lambda A)-\lambda\left(A \circ J_{\lambda}^{B}(I-\lambda A)-A\right)\right](\dot{x}(t)+x(t)),
$$

where $\alpha:[0, \infty) \rightarrow[0, \infty)$ is a Lebesgue measurable function. Observe that the dynamical system (9) is not explicit because $\dot{x}(t)$ appears on both sides of (9). The dynamical system (9) is different from the second order dynamical system considered in ([27] Section 2$)$.

Using the forward discretization $\dot{x}(t) \approx \frac{x_{n+1}-x_{n}}{h}$ on the left-hand side and the backward discretization $\dot{x}(t) \approx \frac{x_{n}-x_{n-1}}{h}$ on the right-hand side of (9), we have

$$
\begin{aligned}
\frac{x_{n+1}-x_{n}}{h}+\alpha_{n} x_{n}= & \alpha_{n}\left[J_{\lambda}^{B}(I-\lambda A)\right. \\
& \left.-\lambda\left(A \circ J_{\lambda}^{B}(I-\lambda A)-A\right)\right]\left(x_{n}+\frac{x_{n}-x_{n-1}}{h}\right) .
\end{aligned}
$$

When $h=1$, (10) becomes

$$
\begin{aligned}
x_{n+1}= & \left(1-\alpha_{n}\right) x_{n}+\alpha_{n}\left[J_{\lambda}^{B}(I-\lambda A)\right. \\
& \left.-\lambda\left(A \circ J_{\lambda}^{B}(I-\lambda A)-A\right)\right]\left(2 x_{n}-x_{n-1}\right) \\
= & \left(1-\alpha_{n}\right) x_{n}+\alpha_{n}\left[J_{\lambda}^{B}(I-\lambda A)\left(2 x_{n}-x_{n-1}\right)\right. \\
& \left.-\lambda\left(A \circ J_{\lambda}^{B}(I-\lambda A)\left(2 x_{n}-x_{n-1}\right)-A\left(2 x_{n}-x_{n-1}\right)\right)\right] .
\end{aligned}
$$

We call (11) the reflected forward-backward-forward method. Taking $w_{n}:=2 x_{n}-x_{n-1}$, $y_{n}:=J_{\lambda}^{B}(I-\lambda A) w_{n}$, then $(11)$ becomes

$$
x_{n+1}=\left(1-\alpha_{n}\right) x_{n}+\alpha_{n}\left(y_{n}-\lambda\left(A y_{n}-A w_{n}\right)\right) .
$$

Our interest is to study (13) in a more general setting $\lambda:=\lambda_{n}$ for solving (1).

The rest of the paper is organized as follows: Next we recall some basic definitions and results. In Section 3 we present and analyze our two forward-backward-forward methods, and then final conclusions are given in Section 4. 


\section{Preliminaries}

We give some lemmas for our analysis.

Lemma 1 (See [2]). The following statements hold in $\mathrm{H}$ :

(i) $\|x+y\|^{2}=\|x\|^{2}+2\langle x, y\rangle+\|y\|^{2}$ for all $x, y \in H$;

(ii) $\|x+y\|^{2} \leq\|x\|^{2}+2\langle y, x+y\rangle$ for all $x, y \in H$

(iii) $\|x+y\|^{2}=2\|x\|^{2}+2\|y\|^{2}-\|x-y\|^{2}, \quad \forall x, y \in H$.

Lemma 2 (Maingé [28]). Let $\left\{\varphi_{n}\right\},\left\{\delta_{n}\right\}$ and $\left\{\theta_{n}\right\}$ be sequences in $[0,+\infty)$ such that

$$
\varphi_{n+1} \leq \varphi_{n}+\theta_{n}\left(\varphi_{n}-\varphi_{n-1}\right)+\delta_{n}, \forall n \geq 1, \sum_{n=1}^{+\infty} \delta_{n}<+\infty,
$$

and there exists a real number $\theta$ with $0 \leq \theta_{n} \leq \theta<1$ for all $n \in \mathbb{N}$. Then the following hold:

(i) $\sum_{n=1}^{+\infty}\left[\varphi_{n}-\varphi_{n-1}\right]_{+}<+\infty$, where $[t]_{+}:=\max \{t, 0\}$;

(ii) there exists $\varphi^{*} \in[0,+\infty)$ such that $\lim _{n \rightarrow \infty} \varphi_{n}=\varphi^{*}$.

Lemma 3 (Opial [29]). Let $C$ be a nonempty set of $H$ and $\left\{x_{n}\right\}$ be a sequence in $H$ such that the following two conditions hold:

(i) for any $x \in C, \lim _{n \rightarrow \infty}\left\|x_{n}-x\right\|$ exists;

(ii) every sequential weak cluster point of $\left\{x_{n}\right\}$ is in $C$.

Then $\left\{x_{n}\right\}$ converges weakly to a point in $C$.

Lemma 4 (Xu [30]). Let $\left\{a_{n}\right\}$ be a sequence of nonnegative real numbers satisfying the following relation:

$$
a_{n+1} \leq\left(1-\alpha_{n}\right) a_{n}+\alpha_{n} \sigma_{n}+\gamma_{n}, n \geq 1,
$$

where

(a) $\left\{\alpha_{n}\right\} \subset[0,1], \sum_{k=1}^{\infty} \alpha_{n}=\infty$;

(b) $\lim \sup \sigma_{n} \leq 0$;

(c) $\gamma_{n} \geq 0(n \geq 1), \sum_{n=1}^{\infty} \gamma_{n}<\infty$.

Then, $a_{n} \rightarrow 0$ as $n \rightarrow \infty$.

Definition 1. A mapping $A: H \rightarrow H$ is called

(a) strongly monotone with modulus $\gamma>0$ on $\mathrm{H}$ if

$$
\langle A x-A y, x-y\rangle \geq \gamma\|x-y\|^{2}, \forall x, y \in H .
$$

In this case, we say that $A$ is $\gamma$-strongly monotone;

(b) monotone on $\mathrm{H}$ if

$$
\langle A x-A y, x-y\rangle \geq 0, \forall x, y \in H
$$

(c) co-coercive on $\mathrm{H}$ if

$$
\langle A x-A y, x-y\rangle \geq \gamma\|A x-A y\|^{2}, \forall x, y \in H ;
$$

(d) Lipschitz continuous on $H$ if there exists a constant $L>0$ such that $\|A x-A y\| \leq L\|x-y\|$ for all $x, y \in H$. 
Definition 2. A multi-valued operator $B: H \rightarrow 2^{H}$ with graph $G(T)=\left\{\left(x, x^{*}\right): x^{*} \in B x\right\}$ is said to be monotone if for any $x, y \in D(T), x^{*} \in B x$ and $y^{*} \in B y$

$$
\left\langle x-y, x^{*}-y^{*}\right\rangle \geq 0
$$

A monotone operator $B$ is said to be maximal if $B=S$ whenever $S: H \rightarrow 2^{H}$ is monotone and $G(B) \subset G(S)$. For more details, see, for instance [31].

Lemma 5 (See [2]). Let $B: H \rightarrow 2^{H}$ be a maximal monotone mapping and $A: H \rightarrow H$ be a mapping. Define a mapping

$$
T_{\lambda} x:=J_{\lambda}^{B}(I-\lambda A)(x), x \in H, \lambda>0 .
$$

Then $F\left(T_{\lambda}\right)=(A+B)^{-1}(0)$, where $F\left(T_{\lambda}\right)$ denotes the set of all fixed points of $T_{\lambda}$.

\section{Our Results}

We give two new Forward-Backward-Forward algorithms and their analysis under suitable conditions. We assume that the following conditions hold for the rest of this paper.

\section{Assumption 1}

(a) Let $B: H \rightarrow 2^{H}$ be a maximal monotone operator; $A: H \rightarrow H$ a monotone and L-Lipschitz continuous.

(b) The solution set $(A+B)^{-1}(0)$ of the inclusion problem (1) is nonempty.

(c) $0<\alpha \leq \alpha_{n} \leq \alpha_{n+1} \leq \frac{1}{2+\delta}:=\varepsilon, \delta>0$.

\section{Remark 1.}

1. Observe that by (15) it is clear that $\lambda_{n+1} \leq \lambda_{n}, \forall n \geq 1$. Moreover, if $A w_{n} \neq A y_{n}$, then

$$
\frac{\mu\left\|w_{n}-y_{n}\right\|}{\left\|A w_{n}-A y_{n}\right\|} \geq \frac{\mu}{L} \frac{\left\|w_{n}-y_{n}\right\|}{\left\|w_{n}-y_{n}\right\|}=\frac{\mu}{L}
$$

and thus $0<\min \left\{\lambda_{1}, \frac{\mu}{L}\right\} \leq \lambda_{n} \forall n \geq 1$. This means that $\lim _{n \rightarrow \infty} \lambda_{n}$ exists. Therefore, $\lim _{n \rightarrow \infty} \lambda_{n}=\lambda>0$.

2. By Lemma 5, it is clear that if $w_{n}=y_{n}$, then $w_{n}$ is a solution of problem (1).

Lemma 6. The sequence $\left\{x_{n}\right\}_{n=1}^{\infty}$ generated by Algorithm 1 is bounded. 
Algorithm 1 Forward-Backward-Forward Algorithm 1

1: Choose parameters as in Assumptions $1(\mathrm{c}), \mu \in(0,1)$ and $\lambda_{1}>0$. Choose arbitrary starting points $x_{0}, x_{1} \in H$. Set $n:=1$.

2: Given the iterates $x_{n}, x_{n-1}$, compute

$$
\left\{\begin{array}{l}
w_{n}=2 x_{n}-x_{n-1} \\
y_{n}=J_{\lambda_{n}}^{B}\left(w_{n}-\lambda_{n} A w_{n}\right)
\end{array}\right.
$$

where

$$
\lambda_{n+1}= \begin{cases}\min \left\{\frac{\mu\left\|w_{n}-y_{n}\right\|}{\left\|A w_{n}-A y_{n}\right\|},\right. & \left.\lambda_{n}\right\}, A w_{n} \neq A y_{n} \\ \lambda_{n}, & \text { otherwise. }\end{cases}
$$

If $w_{n}=y_{n}$, then STOP.

3: Otherwise, compute the next iterate via

$$
x_{n+1}=\left(1-\alpha_{n}\right) x_{n}+\alpha_{n}\left[y_{n}-\lambda_{n}\left(A y_{n}-A w_{n}\right)\right] .
$$

4: Set $n \leftarrow n+1$ and go to 2 .

Proof. Let us define $u_{n}:=y_{n}-\lambda_{n}\left(A y_{n}-A w_{n}\right), n \geq 1$. Then

$$
\begin{aligned}
\left\|u_{n}-p\right\|^{2}= & \left\|y_{n}-\lambda_{n}\left(A y_{n}-A w_{n}\right)-p\right\|^{2} \\
= & \left\|y_{n}-p\right\|^{2}+\lambda_{n}^{2}\left\|A y_{n}-A w_{n}\right\|-2 \lambda_{n}\left\langle y_{n}-p, A y_{n}-A w_{n}\right\rangle \\
= & \left\|w_{n}-p\right\|^{2}+\left\|w_{n}-y_{n}\right\|^{2}+2\left\langle y_{n}-w_{n}, w_{n}-p\right\rangle+\lambda_{n}^{2}\left\|A y_{n}-A w_{n}\right\|^{2} \\
- & 2 \lambda_{n}\left\langle y_{n}-p, A y_{n}-A w_{n}\right\rangle \\
= & \left\|w_{n}-p\right\|^{2}+\left\|w_{n}-y_{n}\right\|^{2}-2\left\langle y_{n}-w_{n}, y_{n}-w_{n}\right\rangle+2\left\langle y_{n}-w_{n}, y_{n}-p\right\rangle \\
& +\lambda_{n}^{2}\left\|A y_{n}-A w_{n}\right\|^{2}-2 \lambda_{n}\left\langle y_{n}-p, A y_{n}-A w_{n}\right\rangle \\
= & \left\|w_{n}-p\right\|^{2}-\left\|w_{n}-y_{n}\right\|^{2}-2\left\langle y_{n}-w_{n}, y_{n}-p\right\rangle+\lambda_{n}^{2}\left\|A y_{n}-A w_{n}\right\|^{2} \\
- & 2 \lambda_{n}\left\langle y_{n}-p, A y_{n}-A w_{n}\right\rangle \\
= & \left\|w_{n}-p\right\|^{2}-\left\|w_{n}-y_{n}\right\|^{2}+\lambda_{n}^{2}\left\|A y_{n}-A w_{n}\right\|^{2} \\
- & 2\left\langle w_{n}-y_{n}-\lambda_{n}\left(A w_{n}-A y_{n}\right), y_{n}-p\right\rangle \\
\leq & \left\|w_{n}-p\right\|^{2}-\left\|w_{n}-y_{n}\right\|^{2}+\frac{\lambda_{n}^{2} \mu^{2}}{\lambda_{n+1}^{2}}\left\|y_{n}-w_{n}\right\|^{2} \\
- & 2\left\langle w_{n}-y_{n}-\lambda_{n}\left(A w_{n}-A y_{n}\right), y_{n}-p\right\rangle \\
= & \left\|w_{n}-p\right\|^{2}-\left(1-\frac{\lambda_{n}^{2} \mu^{2}}{\lambda_{n+1}^{2}}\right)\left\|y_{n}-w_{n}\right\|^{2} \\
- & 2\left\langle w_{n}-y_{n}-\lambda_{n}\left(A w_{n}-A y_{n}\right), y_{n}-p\right\rangle .
\end{aligned}
$$

We now show that

$$
\left\langle w_{n}-y_{n}-\lambda_{n}\left(A w_{n}-A y_{n}\right), y_{n}-p\right\rangle \geq 0 .
$$

From $y_{n}=\left(I+\lambda_{n} B\right)^{-1}\left(w_{n}-\lambda_{n} A w_{n}\right)$, we obtain $w_{n}-\lambda_{n} A w_{n} \in y_{n}+\lambda_{n} B y_{n}$. Noting that $B$ is maximal monotone, we obtain $v_{n} \in B y_{n}$, such that

$$
\left(I-\lambda_{n} A\right) w_{n}=y_{n}+\lambda_{n} v_{n}
$$


Therefore,

$$
v_{n}=\frac{1}{\lambda_{n}}\left(w_{n}-y_{n}-\lambda_{n} A w_{n}\right) .
$$

Furthermore, $0 \in(A+B) p$ and $A y_{n}+v_{n} \in(A+B) y_{n}$. Since $A+B$ is maximal monotone, one has

$$
\left\langle A y_{n}+v_{n}, y_{n}-p\right\rangle \geq 0
$$

Putting (20) into (19), we have

$$
\frac{1}{\lambda_{n}}\left\langle w_{n}-y_{n}-\lambda_{n} A w_{n}+\lambda_{n} A y_{n}, y_{n}-p\right\rangle \geq 0
$$

Thus,

$$
\left\langle w_{n}-y_{n}-\lambda_{n}\left(A w_{n}-A y_{n}\right), y_{n}-p\right\rangle \geq 0 .
$$

This is (18). Using (18) in (17), we get

$$
\left\|u_{n}-p\right\|^{2} \leq\left\|w_{n}-p\right\|^{2}-\left(1-\frac{\lambda_{n}^{2} \mu^{2}}{\lambda_{n+1}^{2}}\right)\left\|y_{n}-w_{n}\right\|^{2} .
$$

Using Algorithm 1, we get

$$
\begin{aligned}
\left\|x_{n+1}-p\right\|^{2}= & \left\|\left(1-\alpha_{n}\right)\left(x_{n}-p\right)+\alpha_{n}\left(u_{n}-p\right)\right\|^{2} \\
= & \left(1-\alpha_{n}\right)\left\|x_{n}-p\right\|^{2}+\alpha_{n}\left\|u_{n}-p\right\|^{2} \\
& -\alpha_{n}\left(1-\alpha_{n}\right)\left\|x_{n}-u_{n}\right\|^{2},
\end{aligned}
$$

which in turn implies that

$$
\begin{aligned}
\left\|x_{n+1}-p\right\|^{2} \leq & \left(1-\alpha_{n}\right)\left\|x_{n}-p\right\|^{2}+\alpha_{n}\left\|w_{n}-p\right\|^{2} \\
& -\alpha_{n}\left(1-\alpha_{n}\right)\left\|x_{n}-u_{n}\right\|^{2} .
\end{aligned}
$$

Note that

$$
x_{n+1}=\left(1-\alpha_{n}\right) x_{n}+\alpha_{n} u_{n}
$$

and this implies

$$
u_{n}-x_{n}=\frac{1}{\alpha_{n}}\left(x_{n+1}-x_{n}\right), \forall n .
$$

Using (24) in (23), we get

$$
\begin{aligned}
\left\|x_{n+1}-p\right\|^{2} \leq & \left(1-\alpha_{n}\right)\left\|x_{n}-p\right\|^{2}+\alpha_{n}\left\|w_{n}-p\right\|^{2} \\
& -\frac{\left(1-\alpha_{n}\right)}{\alpha_{n}}\left\|x_{n+1}-x_{n}\right\|^{2}
\end{aligned}
$$

Additionally, by Lemma 1 (iii),

$$
\begin{aligned}
\left\|w_{n}-p\right\|^{2}= & \left\|2 x_{n}-x_{n-1}-p\right\|^{2} \\
= & \left\|\left(x_{n}-p\right)+\left(x_{n}-x_{n-1}\right)\right\|^{2} \\
= & 2\left\|x_{n}-p\right\|^{2}-\left\|x_{n-1}-p\right\|^{2} \\
& +2\left\|x_{n}-x_{n-1}\right\|^{2} .
\end{aligned}
$$


Using (26) in (25):

$$
\begin{aligned}
\left\|x_{n+1}-p\right\|^{2} \leq & \left(1-\alpha_{n}\right)\left\|x_{n}-p\right\|^{2}+2 \alpha_{n}\left\|x_{n}-p\right\|^{2} \\
& -\alpha_{n}\left\|x_{n-1}-p\right\|^{2}+2 \alpha_{n}\left\|x_{n}-x_{n-1}\right\|^{2} \\
& -\frac{1-\alpha_{n}}{\alpha_{n}}\left\|x_{n+1}-x_{n}\right\|^{2} \\
= & \left(1+\alpha_{n}\right)\left\|x_{n}-p\right\|^{2}-\alpha_{n}\left\|x_{n-1}-p\right\|^{2} \\
& +2 \alpha_{n}\left\|x_{n}-x_{n-1}\right\|^{2} \\
& -\frac{1-\alpha_{n}}{\alpha_{n}}\left\|x_{n+1}-x_{n}\right\|^{2} .
\end{aligned}
$$

Define

$$
\Gamma_{n}:=\left\|x_{n}-p\right\|^{2}-\alpha_{n}\left\|x_{n-1}-p\right\|^{2}+2 \alpha_{n}\left\|x_{n}-x_{n-1}\right\|^{2}, n \geq 1 .
$$

Since $\alpha_{n} \leq \alpha_{n+1}$, we have

$$
\begin{aligned}
\Gamma_{n+1}-\Gamma_{n}= & \left\|x_{n+1}-p\right\|^{2}-\left(1+\alpha_{n+1}\right)\left\|x_{n}-p\right\|^{2} \\
& +\alpha_{n}\left\|x_{n-1}-p\right\|^{2}+2 \alpha_{n+1}\left\|x_{n+1}-x_{n}\right\|^{2} \\
& -2 \alpha_{n}\left\|x_{n}-x_{n-1}\right\|^{2} \\
\leq & \left\|x_{n+1}-p\right\|^{2}-\left(1+\alpha_{n}\right)\left\|x_{n}-p\right\|^{2}+\alpha_{n}\left\|x_{n-1}-p\right\|^{2} \\
& +2 \alpha_{n+1}\left\|x_{n+1}-x_{n}\right\|^{2} \\
& -2 \alpha_{n}\left\|x_{n}-x_{n-1}\right\|^{2} .
\end{aligned}
$$

Now, using (27) in (28), one gets

$$
\begin{aligned}
\Gamma_{n+1}-\Gamma_{n} & \leq-\frac{1-\alpha_{n}}{\alpha_{n}}\left\|x_{n+1}-x_{n}\right\|^{2}+2 \alpha_{n+1}\left\|x_{n+1}-x_{n}\right\|^{2} \\
& =-\left(\frac{1-\alpha_{n}}{\alpha_{n}}-2 \alpha_{n+1}\right)\left\|x_{n+1}-x_{n}\right\|^{2} .
\end{aligned}
$$

By condition (c) of Assumption 1, one gets

$$
\begin{aligned}
\frac{1-\alpha_{n}}{\alpha_{n}}-2 \alpha_{n+1} & =\frac{1}{\alpha_{n}}-1-2 \alpha_{n+1} \\
& \geq 2+\delta-1-\frac{2}{2+\delta} \\
& =\delta+\frac{\delta}{2+\delta} \geq \delta .
\end{aligned}
$$

Using (30) in (29), we have

$$
\Gamma_{n+1}-\Gamma_{n} \leq-\delta\left\|x_{n+1}-x_{n}\right\|^{2} .
$$

Therefore, $\left\{\Gamma_{n}\right\}$ is non-increasing. Similarly,

$$
\begin{aligned}
\Gamma_{n} & =\left\|x_{n}-p\right\|^{2}-\alpha_{n}\left\|x_{n-1}-p\right\|^{2}+2 \alpha_{n}\left\|x_{n}-x_{n-1}\right\|^{2} \\
& \geq\left\|x_{n}-p\right\|^{2}-\alpha_{n}\left\|x_{n-1}-p\right\|^{2} .
\end{aligned}
$$

Note that

$$
\alpha_{n}<\frac{1}{2+\delta}=\epsilon<1
$$


From (32), we have

$$
\begin{aligned}
\left\|x_{n}-p\right\|^{2} & \leq \alpha_{n}\left\|x_{n-1}-p\right\|^{2}+\Gamma_{n} \\
& \leq \epsilon\left\|x_{n-1}-p\right\|^{2}+\Gamma_{1} \\
& \vdots \\
& \leq \epsilon^{n}\left\|x_{0}-p\right\|^{2}+\left(1+\cdots+\epsilon^{n-1}\right) \Gamma_{1} \\
& \leq \epsilon^{n}\left\|x_{0}-p\right\|^{2}+\frac{\Gamma_{1}}{1-\epsilon} .
\end{aligned}
$$

\section{Consequently,}

$$
\begin{aligned}
\Gamma_{n+1}= & \left\|x_{n+1}-p\right\|^{2}-\alpha_{n+1}\left\|x_{n}-p\right\|^{2} \\
& +2 \alpha_{n+1}\left\|x_{n+1}-x_{n}\right\|^{2} \\
\geq & -\alpha_{n+1}\left\|x_{n}-p\right\|^{2}
\end{aligned}
$$

and this means from (33) that

$$
\begin{aligned}
-\Gamma_{n+1} & \leq \alpha_{n+1}\left\|x_{n}-p\right\|^{2} \\
& \leq \epsilon\left\|x_{n}-p\right\|^{2} \\
& \vdots \\
& \leq \epsilon^{n+1}\left\|x_{0}-p\right\|^{2}+\frac{\epsilon \Gamma_{1}}{1-\epsilon} .
\end{aligned}
$$

By (31) and (34), we get

$$
\begin{aligned}
\delta \sum_{n=1}^{k}\left\|x_{n+1}-x_{n}\right\|^{2} & \leq \Gamma_{1}-\Gamma_{k+1} \\
& \leq \epsilon^{k+1}\left\|x_{0}-p\right\|^{2}+\frac{\Gamma_{1}}{1-\epsilon} .
\end{aligned}
$$

This implies

$$
\sum_{n=1}^{\infty}\left\|x_{n+1}-x_{n}\right\|^{2} \leq \frac{\Gamma_{1}}{\delta(1-\epsilon)}<+\infty .
$$

Therefore, $\lim _{n \rightarrow \infty}\left\|x_{n+1}-x_{n}\right\|=0$. Additionally, from (16), we get

$$
\left\|w_{n}-x_{n}\right\|=\left\|x_{n}-x_{n-1}\right\| \rightarrow 0, n \rightarrow \infty .
$$

From (27), we get

$$
\begin{aligned}
\left\|x_{n+1}-p\right\|^{2} \leq & \left(1+\alpha_{n}\right)\left\|x_{n}-p\right\|^{2}-\alpha_{n}\left\|x_{n-1}-p\right\|^{2} \\
& +2\left\|x_{n}-x_{n-1}\right\|^{2} .
\end{aligned}
$$

Using Lemma 2 in (38) (noting (36)), we get

$$
\lim _{n \rightarrow \infty}\left\|x_{n}-p\right\|^{2}=l<\infty .
$$


Hence, $\left\{\left\|x_{n}-p\right\|\right\}$ is bounded. Therefore $\left\{x_{n}\right\}$ is bounded.

Theorem 1. $\left\{x_{n}\right\}_{n=1}^{\infty}$ generated by Algorithm 1 converges weakly to a point in $(A+B)^{-1}(0)$.

Proof. By (24), we have

$$
\begin{aligned}
\left\|u_{n}-x_{n}\right\| & =\frac{1}{\alpha_{n}}\left\|x_{n+1}-x_{n}\right\| \\
& \leq \frac{1}{\alpha}\left\|x_{n+1}-x_{n}\right\| \rightarrow 0, n \rightarrow \infty
\end{aligned}
$$

Therefore,

$$
\left\|w_{n}-u_{n}\right\| \leq\left\|u_{n}-x_{n}\right\|+\left\|w_{n}-x_{n}\right\| \rightarrow 0, n \rightarrow \infty .
$$

Now, $\left\{w_{n}\right\}$ and $\left\{u_{n}\right\}$ are bounded by the boundedness of $\left\{x_{n}\right\}$. Hence, there exists $M>0$ such that (noting (21))

$$
\begin{aligned}
\left(1-\frac{\lambda_{n}^{2} \mu^{2}}{\lambda_{n+1}^{2}}\right)\left\|y_{n}-w_{n}\right\|^{2} & \leq\left\|w_{n}-p\right\|^{2}-\left\|u_{n}-p\right\|^{2} \\
& =\left(\left\|w_{n}-p\right\|+\left\|u_{n}-p\right\|\right)\left(\left\|w_{n}-p\right\|-\left\|u_{n}-p\right\|\right) \\
& \leq M\left\|w_{n}-u_{n}\right\| \rightarrow 0, n \rightarrow \infty
\end{aligned}
$$

Therefore,

$$
\left\|y_{n}-w_{n}\right\| \rightarrow 0, n \rightarrow \infty .
$$

Additionally, by the boundedness of $\left\{x_{n}\right\}$, there exists subsequence $\left\{x_{n_{j}}\right\}$ such that $x_{n_{j}} \rightarrow z \in H$.

Let $(v, u) \in \operatorname{Graph}(A+B)$. Thus, $u-A v \in B v$ and so $y_{n_{j}}=\left(I+\lambda_{n_{j}} B\right)^{-1}\left(I-\lambda_{n_{j}} A\right) w_{n_{j}}$. Hence, $\left(I-\lambda_{n_{j}} A\right) w_{n_{j}} \in\left(I+\lambda_{n_{j}} B\right) y_{n_{j}}$, which turns to

$$
\frac{1}{\lambda_{n_{j}}}\left(w_{n_{j}}-y_{n_{j}}-\lambda_{n_{j}} A w_{n_{j}}\right) \in B y_{n_{j}}
$$

$B$ is maximal monotone, giving

$$
\left\langle v-y_{n_{j}}, u-A v-\frac{1}{\lambda_{n_{j}}}\left(w_{n_{j}}-y_{n_{j}}-\lambda_{n_{j}} A w_{n_{j}}\right)\right\rangle \geq 0 .
$$

Thus,

$$
\begin{aligned}
\left\langle v-y_{n_{j}}, u\right\rangle \geq & \left\langle v-y_{n_{j}}, A v-\frac{1}{\lambda_{n_{j}}}\left(w_{n_{j}}-y_{n_{j}}-\lambda_{n_{j}} A w_{n_{j}}\right)\right\rangle \\
= & \left\langle v-y_{n_{j}}, A v-A w_{n_{j}}\right\rangle+\left\langle v-y_{n_{j}}, \frac{1}{\lambda_{n_{j}}}\left(w_{n_{j}}-y_{n_{j}}\right)\right\rangle \\
= & \left\langle v-y_{n_{j}}, A v-A y_{n_{j}}\right\rangle+\left\langle v-y_{n_{j}}, A y_{n_{j}}-A w_{n_{j}}\right\rangle \\
& +\left\langle v-y_{n_{j}}, \frac{1}{\lambda_{n_{j}}}\left(w_{n_{j}}-y_{n_{j}}\right)\right\rangle \\
\geq & \left\langle v-y_{n_{j}}, A y_{n_{j}}-A w_{n_{j}}\right\rangle+\left\langle v-y_{n_{n}}, \frac{1}{\lambda_{n_{j}}}\left(w_{n_{j}}-y_{n_{j}}\right)\right\rangle .
\end{aligned}
$$


Since $\lim _{n \rightarrow \infty}\left\|w_{n}-y_{n}\right\|=0$ and $A$ is Lipschitz continuous, we get $\lim _{j \rightarrow \infty}\left\|A w_{n_{j}}-A y_{n_{j}}\right\|=0$. Furthermore, since $0<\min \left\{\lambda_{1}, \frac{\mu}{L}\right\} \leq \lambda_{n} \forall n \geq 1$, we get from (43) that

$$
\langle v-z, u\rangle=\lim _{j \rightarrow \infty}\left\langle v-y_{n_{j}}, u\right\rangle \geq 0
$$

This implies by the maximal monotonicity of $A+B$ (see ([2] Corollary 24.4(i))) that $0 \in(A+B) z$. Thus $0 \in(A+B)^{-1}(0)$.

Since by (39), we have that $\lim _{n \rightarrow \infty}\left\|x_{n}-z\right\|$ exists. Hence, Opial's lemma 3 shows that $\left\{x_{n}\right\}$ converges weakly to a point in $(A+B)^{-1}(0)$. This completes the proof.

If $A$ is strongly monotone and Lipschitz continuous on $H$, we show that $\left\{x_{n}\right\}_{n=1}^{\infty}$ converges strongly in Algorithm 1. Note that in this case the splitting operator $T_{\lambda}$ in Lemma 5 is a contraction mapping and hence $(A+B)^{-1}(0)$ is a singleton.

Theorem 2. Suppose A is strongly monotone and Lipschitz continuous on $H$. Then $\left\{x_{n}\right\}_{n=1}^{\infty}$ generated Algorithm 1 converges strongly to the unique point in $(A+B)^{-1}(0)$.

Proof. Take unique point $p \in(A+B)^{-1}(0)$. From the definition of $y_{n}$, there exists $v_{n} \in B y_{n}$, such that

$$
y_{n}+\lambda_{n} v_{n}=w_{n}-\lambda_{n} A w_{n} .
$$

From $0 \in A p+B p$, there exists $v^{*} \in B p$, such that $A p+v^{*}=0$. Given that $u_{n}=y_{n}-\lambda_{n}\left(A y_{n}-A w_{n}\right)$, $n \geq 1$, we have

$$
\begin{aligned}
\left\|w_{n}-p\right\|^{2}= & \left\|w_{n}-y_{n}+y_{n}-u_{n}+u_{n}-p\right\|^{2} \\
= & \left\|w_{n}-y_{n}\right\|^{2}+\left\|y_{n}-u_{n}\right\|^{2}+\left\|u_{n}-p\right\|^{2} \\
& +2\left\langle w_{n}-y_{n}, y_{n}-p\right\rangle+2\left\langle y_{n}-u_{n}, u_{n}-p\right\rangle \\
= & \left\|w_{n}-y_{n}\right\|^{2}-\left\|y_{n}-u_{n}\right\|^{2}+\left\|u_{n}-p\right\|^{2} \\
& +2\left\langle w_{n}-u_{n}, y_{n}-p\right\rangle \\
= & \left\|w_{n}-y_{n}\right\|^{2}-\lambda_{n}^{2}\left\|A w_{n}-A y_{n}\right\|^{2}+\left\|u_{n}-p\right\|^{2} \\
& +2 \lambda_{n}\left\langle A y_{n}+v_{n}, y_{n}-p\right\rangle \\
= & \left\|w_{n}-y_{n}\right\|^{2}-\lambda_{n}^{2}\left\|A w_{n}-A y_{n}\right\|^{2}+\left\|u_{n}-p\right\|^{2} \\
& +2 \lambda_{n}\left\langle A y_{n}-A p+v_{n}-v^{*}, y_{n}-p\right\rangle .
\end{aligned}
$$

Since $A+B$ is strongly monotone, there exists $\eta>0$ such that

$$
\left\langle A y_{n}-A p+v_{n}-v^{*}, y_{n}-p\right\rangle \geq \eta\left\|y_{n}-p\right\|^{2} .
$$

Now, from (44) and (45) we have

$$
\begin{aligned}
\left\|u_{n}-p\right\|^{2} \leq & \left\|w_{n}-p\right\|^{2}-\left\|w_{n}-y_{n}\right\|^{2}+\lambda_{n}^{2}\left\|A w_{n}-A y_{n}\right\|^{2} \\
& -2 \lambda_{n} \eta\left\|y_{n}-p\right\|^{2} \\
\leq & \left\|w_{n}-p\right\|^{2}-\left(1-\frac{\lambda_{n}^{2} \mu^{2}}{\lambda_{n+1}^{2}}\right)\left\|w_{n}-y_{n}\right\|^{2} \\
& -2 \lambda_{n} \eta\left\|y_{n}-p\right\|^{2} .
\end{aligned}
$$


Since $\lim _{n \rightarrow \infty} \lambda_{n}=\lambda$ and the sequence $\left\{\lambda_{n}\right\}$ is monotonically decreasing, we have $\lambda_{n} \geq \lambda \forall n \geq 1$. Let $\rho$ be a fixed number in the interval $(\mu, 1)$. Additionally, since $\lim _{n \rightarrow \infty} \frac{\lambda_{n} \mu}{\lambda_{n+1}}=\mu<\rho$, there exists $N>0$ such that $\frac{\lambda_{n} \mu}{\lambda_{n+1}}<\rho \forall n \geq N$. So, $\forall n \geq N$, we have

$$
\lambda_{n} \geq \lambda, \frac{\lambda_{n} \mu}{\lambda_{n+1}}<\rho
$$

Plugging (26) and (47) in (46), we have $\forall n \geq N$,

$$
\begin{aligned}
\left\|u_{n}-p\right\|^{2} \leq & \left\|w_{n}-p\right\|^{2}-\left(1-\rho^{2}\right)\left\|w_{n}-y_{n}\right\|^{2}-2 \lambda \eta\left\|y_{n}-p\right\|^{2} \\
= & \left\|2 x_{n}-x_{n-1}-p\right\|^{2}-\left(1-\rho^{2}\right)\left\|w_{n}-y_{n}\right\|^{2} \\
& -2 \lambda \eta\left\|y_{n}-p\right\|^{2} \\
= & 2\left\|x_{n}-p\right\|^{2}-\left\|x_{n-1}-p\right\|^{2}+2\left\|x_{n}-x_{n-1}\right\|^{2} \\
& -\left(1-\rho^{2}\right)\left\|w_{n}-y_{n}\right\|^{2}-2 \lambda \eta\left\|y_{n}-p\right\|^{2} .
\end{aligned}
$$

Consequently,

$$
\begin{aligned}
\left\|x_{n+1}-p\right\|^{2} \leq & \left(1-\alpha_{n}\right)\left\|x_{n}-p\right\|^{2}+\alpha_{n}\left\|u_{n}-x^{*}\right\|^{2} \\
\leq & \left(1-\alpha_{n}\right)\left\|x_{n}-p\right\|^{2}+\alpha_{n}\left[2\left\|x_{n}-p\right\|^{2}-\left\|x_{n-1}-p\right\|^{2}\right. \\
& +2\left\|x_{n}-x_{n-1}\right\|^{2}-\left(1-\rho^{2}\right)\left\|w_{n}-y_{n}\right\|^{2} \\
& \left.-2 \lambda \eta\left\|y_{n}-p\right\|^{2}\right] \\
\leq & \left(1+\alpha_{n}\right)\left\|x_{n}-p\right\|^{2}-\alpha_{n}\left\|x_{n-1}-p\right\|^{2} \\
& +2 \alpha_{n}\left\|x_{n}-x_{n-1}\right\|^{2}-\alpha_{n}\left(1-\rho^{2}\right)\left\|w_{n}-y_{n}\right\|^{2} \\
& -2 \alpha_{n} \lambda \eta\left\|y_{n}-p\right\|^{2} .
\end{aligned}
$$

Therefore

$$
\begin{aligned}
2 \alpha \lambda \eta\left\|y_{n}-p\right\|^{2} \leq & \left\|x_{n}-p\right\|^{2}-\left\|x_{n+1}-p\right\|^{2} \\
& +\alpha_{n}\left(\left\|x_{n}-p\right\|^{2}-\left\|x_{n-1}-p\right\|^{2}\right)+2\left\|x_{n}-x_{n-1}\right\|^{2} .
\end{aligned}
$$

So,

$$
\begin{aligned}
2 \alpha \lambda \eta \sum_{k=N}^{n}\left\|y_{k}-p\right\|^{2} \leq & \left\|x_{N}-p\right\|^{2}-\left\|x_{n+1}-p\right\|^{2} \\
& +\alpha_{n}\left\|x_{n}-p\right\|^{2}-\alpha_{N-1}\left\|x_{N-1}-p\right\|^{2} \\
& +2 \sum_{k=N}^{n}\left\|x_{k}-x_{k-1}\right\|^{2} .
\end{aligned}
$$

Since $\left\{x_{n}\right\}$ is bounded by Lemma 6 and $\sum_{k=N}^{\infty}\left\|x_{k}-x_{k-1}\right\|^{2}<\infty$ by (36), we obtain $\sum_{k=N}^{\infty}\left\|y_{k}-p\right\|^{2}<\infty$. Hence $\lim _{n \rightarrow \infty}\left\|y_{n}-p\right\|=0$. Consequently, we get

$$
\begin{aligned}
\left\|x_{n}-p\right\| & \leq\left\|x_{n}-y_{n}\right\|+\left\|y_{n}-p\right\| \\
& \leq\left\|x_{n}-w_{n}\right\|+\left\|w_{n}-y_{n}\right\|+\left\|y_{n}-p\right\| \rightarrow 0
\end{aligned}
$$


as $n \rightarrow \infty$. This concludes the proof.

\section{Remark 2.}

1. Observe that the convergence Theorems 1 and 2 assume that the mapping $A$ is monotone and Lipschitz continues. In case that the Lipschitz constant $L$ of $A$ is known or can be easily evaluated, then one can choose the step-sizes $\lambda_{n}$ in Algorithm 1, as follows, and the convergence theorems remain valid.

$$
0<a \leq \lambda_{n} \leq b<\frac{1}{L}
$$

This and our adaptive step-size rule for determining $\left\{\lambda_{n}\right\}$ are quite flexible and general so extend several related results in the literature-e.g., in [22-24,32,33].

2. In case we incorporate a general inertial term $w_{n}=x_{n}+\theta\left(x_{n}-x_{n-1}\right)$ for $\theta \in[0,1)$ (not necessarily 1 ), then Lemma 6 and Theorems 1 and 2 still hold. Moreover, with this general term and under $\eta$-strongly monotonicity and L-Lipschitz continuity we are able to present linear convergence of the next algorithm.

Theorem 3. Suppose that $A$ is $\eta$-strongly monotone and L-Lipschitz continuous on $H$. Then $\left\{x_{n}\right\}_{n=1}^{\infty}$ generated by Algorithm 2 converges linearly to the unique point in $(A+B)^{-1}(0)$.

\section{Algorithm 2 Forward-Backward-Forward Algorithm 2}

1: Choose the step-sizes $\left\{\lambda_{n}\right\}$ as in (50), define $\sigma:=\min \left\{1-b^{2} L^{2}, 2 a \eta\right\} \in(0,1)$ and choose $\theta \in\left[0, \frac{\sigma}{2-\sigma}\right)$ and $\alpha \in\left(0, \frac{1}{2}\right)$. Choose arbitrary starting points $x_{0}, x_{1} \in H$ and set $n:=1$.

2: Given $x_{n}, x_{n-1}$, compute

$$
\left\{\begin{array}{l}
w_{n}=x_{n}+\theta\left(x_{n}-x_{n-1}\right) \\
y_{n}=J_{\lambda_{n}}^{B}\left(w_{n}-\lambda_{n} A w_{n}\right)
\end{array}\right.
$$

If $w_{n}-y_{n}=0$, then STOP.

3: Otherwise, Compute

$$
x_{n+1}=(1-\alpha) x_{n}+\alpha\left[y_{n}-\lambda_{n}\left(A y_{n}-A w_{n}\right)\right] .
$$

4: Set $n \leftarrow n+1$ and go to 2 .

Proof. Following lines of arguments in obtaining (44) and (45), one can show that

$$
\begin{aligned}
\left\|u_{n}-p\right\|^{2} \leq & \left\|w_{n}-p\right\|^{2}-\left\|w_{n}-y_{n}\right\|^{2}+\lambda_{n}^{2}\left\|A w_{n}-A y_{n}\right\|^{2} \\
& -2 \lambda_{n} \eta\left\|y_{n}-p\right\|^{2} \\
\leq & \left\|w_{n}-p\right\|^{2}-\left(1-\lambda_{n}^{2} L^{2}\right)\left\|w_{n}-y_{n}\right\|^{2}-2 \lambda_{n} \eta\left\|y_{n}-p\right\|^{2} \\
\leq & \left\|w_{n}-p\right\|^{2}-\left(1-b^{2} L^{2}\right)\left\|w_{n}-y_{n}\right\|^{2}-2 a \eta\left\|y_{n}-p\right\|^{2} \\
\leq & \left\|w_{n}-p\right\|^{2}-\min \left\{1-b^{2} L^{2}, 2 a \eta\right\}\left(\left\|w_{n}-y_{n}\right\|^{2}+\left\|y_{n}-p\right\|^{2}\right) \\
\leq & \left\|w_{n}-p\right\|^{2}-\frac{1}{2} \min \left\{1-b^{2} L^{2}, 2 a \eta\right\}\left\|w_{n}-p\right\|^{2} \\
= & \zeta^{2}\left\|w_{n}-p\right\|^{2},
\end{aligned}
$$




$$
\begin{aligned}
\zeta^{2}=\left[1-\frac{1}{2} \min \left\{1-b^{2} L^{2}, 2 a \eta\right\}\right] & (0,1) . \text { Consequently, } \\
\left\|x_{n+1}-p\right\|^{2}= & (1-\alpha)\left\|x_{n}-p\right\|^{2}+\alpha\left\|u_{n}-p\right\|^{2}-\alpha(1-\alpha)\left\|x_{n}-u_{n}\right\|^{2} \\
\leq & (1-\alpha)\left\|x_{n}-p\right\|^{2}+\alpha \zeta^{2}\left\|w_{n}-p\right\|^{2}-\frac{1-\alpha}{\alpha}\left\|x_{n+1}-x_{n}\right\|^{2} \\
= & (1-\alpha)\left\|x_{n}-p\right\|^{2}+\alpha \zeta^{2}\left[(1+\theta)\left\|x_{n}-p\right\|^{2}-\theta\left\|x_{n-1}-p\right\|^{2}\right. \\
& \left.+\theta(1+\theta)\left\|x_{n}-x_{n-1}\right\|^{2}\right]-\frac{1-\alpha}{\alpha}\left\|x_{n+1}-x_{n}\right\|^{2} \\
= & \left(1-\alpha\left(1-\zeta^{2}(1+\theta)\right)\right)\left\|x_{n}-p\right\|^{2}-\alpha \zeta^{2} \theta\left\|x_{n-1}-p\right\|^{2} \\
& +\alpha \zeta^{2} \theta(1+\theta)\left\|x_{n}-x_{n-1}\right\|^{2}-\frac{1-\alpha}{\alpha}\left\|x_{n+1}-x_{n}\right\|^{2} .
\end{aligned}
$$

Since $\alpha<\frac{1}{2}$, we therefore have

$$
\begin{aligned}
\left\|x_{n+1}-p\right\|^{2}+\left\|x_{n+1}-x_{n}\right\|^{2} \leq & \left\|x_{n+1}-p\right\|^{2}+\frac{1-\alpha}{\alpha}\left\|x_{n+1}-x_{n}\right\|^{2} \\
\leq & \left(1-\alpha\left(1-\zeta^{2}(1+\theta)\right)\right)\left\|x_{n}-p\right\|^{2} \\
& +\alpha \zeta^{2} \theta(1+\theta)\left\|x_{n}-x_{n-1}\right\|^{2} .
\end{aligned}
$$

Observe that $0<1-\alpha\left(1-\zeta^{2}(1+\theta)\right)<1$ since $\theta \in\left[0, \frac{\sigma}{2-\sigma}\right)$. We obtain from (55) that

$$
\begin{aligned}
& \left\|x_{n+1}-p\right\|^{2}+\left\|x_{n+1}-x_{n}\right\|^{2} \leq\left(1-\alpha\left(1-\zeta^{2}(1+\theta)\right)\right)\left[\left\|x_{n}-p\right\|^{2}\right. \\
& \left.+\frac{\alpha \zeta^{2} \theta(1+\theta)}{1-\alpha\left(1-\zeta^{2}(1+\theta)\right)}\left\|x_{n}-x_{n-1}\right\|^{2}\right] \\
\leq & \left(1-\alpha\left(1-\zeta^{2}(1+\theta)\right)\right)\left[\left\|x_{n}-p\right\|^{2}+\left\|x_{n}-x_{n-1}\right\|^{2}\right],
\end{aligned}
$$

where $\frac{\alpha \zeta^{2} \theta(1+\theta)}{1-\alpha\left(1-\zeta^{2}(1+\theta)\right)}<1$ since $\alpha<\frac{1}{2}<\frac{1}{1-\zeta^{2}(1+\theta)(1-\theta)}$.

Denote $b_{n}:=\left\|x_{n}-p\right\|^{2}+\left\|x_{n}-x_{n-1}\right\|^{2}$. Then (56) implies

$$
b_{n+1} \leq\left(1-\alpha\left(1-\zeta^{2}(1+\theta)\right)\right)^{n} b_{1} .
$$

Therefore,

$$
\left\|x_{n+1}-p\right\|^{2} \leq b_{n+1} \leq\left(1-\alpha\left(1-\zeta^{2}(1+\theta)\right)\right)^{n} b_{1} .
$$

This concludes the proof.

We give some remarks about the contributions of our proposed methods and the consequent improvements over some related methods in the literature.

Remark 3. (a) The proposed methods in [22,23] use a fixed constant step size which depends on the Lipschitz constant of the forward operator $A$. This approach is quite restrictive and has limited applications since the Lipschitz constant or an estimate of it must be known before the methods in [22,23] can be applied. Our proposed method in Algorithm 1 uses a self-adaptive step size in (15), which is more applicable and without recourse to finding the Lipschitz constant or an estimate of it.

(b) In our proposed methods in this paper, the forward-backward $J_{\lambda_{n}}^{B}\left(I-\lambda_{n} A\right)$ acted on the reflection $2 x_{n}-$ $x_{n-1}$, (i.e., there is $\left.J_{\lambda_{n}}^{B}\left(I-\lambda_{n} A\right)\left(2 x_{n}-x_{n-1}\right)\right)$ which speeds up the acceleration of our proposed methods since 
$x=J_{\lambda_{n}}^{B}\left(I-\lambda_{n} A\right) x$ solves the inclusion problem (1). This is not the case in the proposed methods in [22-24]. In these methods, $J_{\lambda_{n}}^{B}\left(I-\lambda_{n} A\right)$ does not act on the reflection $2 x_{n}-x_{n-1}$.

(c) We give a linear convergence rate in Theorem 3. No linear convergence for the proposed methods in $[22,23]$ is given.

\section{Conclusions}

In this work, we study the Tseng-type algorithm with reflection step for solving monotone inclusion in real Hilbert spaces. We propose two variants and their weak and strong convergence results under suitable conditions, as well as convergence rate for stronger assumptions. Our contributions in this paper show that we can modify the Tseng algorithm with extrapolation term $x_{n+1}=x_{n}+\theta\left(x_{n}-x_{n-1}\right)$ with $\theta=1$ and obtain convergence analysis. This approach has not been considered before in the literature. Our work generalizes and extends some related results in the literature, such as in [10,22-24]. Some of the continuing projects that can be studied further are splitting algorithms for finding a zero of the sum of three monotone operators in which two are maximal monotone and the third is Lipschitz continuous—e.g., in [16,33].

Author Contributions: Analysis, Y.S. and A.G.; Investigation, Y.S.; Methodology, Y.S.; Visualization, A.G.; Writing —original draft, Y.S.; Writing-review and editing, A.G. All authors have read and agreed to the published version of the manuscript.

Funding: This research received no external funding.

Conflicts of Interest: The authors declare no conflict of interest.

\section{References}

1. Aoyama, K.; Kimura, Y.; Takahashi, W. Maximal monotone operators and maximal monotone functions for equilibrium problems. J. Convex Anal. 2008, 15, 395-409.

2. Bauschke, H.H.; Combettes, P.L. Convex Analysis and Monotone Operator Theory in Hilbert Spaces, CMS Books in Mathematics; Springer: New York, NY, USA, 2011.

3. Chen, G.H.-G.; Rockafellar, R.T. Convergence rates in forward-backward splitting. SIAM J. Optim. 1997, 7, 421-444. [CrossRef]

4. Combettes, P.; Wajs, V.R. Signal recovery by proximal forward-backward splitting. Multiscale Model. Simul. 2005, 4, 1168-1200. [CrossRef]

5. Lions, P.L.; Mercier, B. Splitting algorithms for the sum of two nonlinear operators. SIAM J. Numer. Anal. 1979, 16, 964-979. [CrossRef]

6. Moudafi, A.; Thera, M. Finding a zero of the sum of two maximal monotone operators. J. Optim. Theory Appl. 1997, 94, 425-448. [CrossRef]

7. Passty, G.B. Ergodic convergence to a zero of the sum of monotone operators in Hilbert spaces. J. Math. Anal. Appl. 1979, 72, 383-390. [CrossRef]

8. Peaceman, D.H.; Rachford, H.H. The numerical solutions of parabolic and elliptic differential equations. J. Soc. Indust. Appl. Math. 1955, 3, 28-41. [CrossRef]

9. Beck, A.; Teboulle, M. Gradient-Based Algorithms with Applications to Signal Recovery Problems. In Convex Optimization in Signal Processing and Communications; Yonina, E., Daniel, P., Eds.; Cambridge University Press: Cambridge, UK, 2009.

10. Tseng, P. A modified forward-backward splitting method for maximal monotone mappings. SIAM J. Control Optim. 2000, 38, 431-446. [CrossRef]

11. Alves, M.M.; Geremia, M. Iteration complexity of an inexact Douglas-Rachford method and of a Douglas-Rachford-Tseng's F-B four-operator splitting method for solving monotone inclusions. Numer. Algorithms 2019, 82, 263-295. [CrossRef]

12. Boț, R.I.; Csetnek, E.R. An inertial Tseng's type proximal algorithm for nonsmooth and nonconvex optimization problems. J. Optim. Theory Appl. 2016, 171, 600-616. [CrossRef] 
13. Cholamjiak, W.; Cholamjiak, P.; Suantai, S. An inertial forward-backward splitting method for solving inclusion problems in Hilbert spaces. J. Fixed Point Theory Appl. 2018, 20, 42. [CrossRef]

14. Gibali, A.; Thong, D.V. Tseng type methods for solving inclusion problems and its applications. Calcolo 2018, 55, 49. [CrossRef]

15. Khatibzadeh, H.; Moroşanu, G.; Ranjbar, S. A splitting method for approximating zeros of the sum of two monotone operators. J. Nonlinear Convex Anal. 2017, 18, 763-776.

16. Latafat, P.; Patrinos, P. Asymmetric forward-backward-adjoint splitting for solving monotone inclusions involving three operators. Comput. Optim. Appl. 2017, 68, 57-93. [CrossRef]

17. Shehu, Y. Convergence results of forward-backward algorithms for sum of monotone operators in Banach spaces. Results Math. 2019, 74, 138. [CrossRef]

18. Shehu, Y.; Cai, G. Strong convergence result of forward-backward splitting methods for accretive operators in Banach spaces with applications. Rev. R. Acad. Cienc. Exactas Fís. Nat. Ser. A Math. RACSAM 2018, 112, 71-87. [CrossRef]

19. Thong, D.V.; Vuong, P.T. Modified Tseng's extragradient methods for solving pseudo-monotone variational inequalities. Optimization 2019, 68, 2203-2222. [CrossRef]

20. Thong, D.V.; Vinh, N. The Inertial methods for fixed point problems and zero point problems of the sum of two monotone mappings. Optimization 2019, 68, 1037-1072. [CrossRef]

21. Wang, Y.; Wang, F. Strong convergence of the forward-backward splitting method with multiple parameters in Hilbert spaces. Optimization 2018, 67, 493-505. [CrossRef]

22. Malitsky, Y.; Tam, M.K. A Forward-Backward splitting method for monotone inclusions without cocoercivity. arXiv 2020, arXiv:1808.04162.

23. Csetnek, E.R.; Malitsky, Y.; Tam, M.K. Shadow Douglas-Rachford Splitting for Monotone Inclusions. Appl. Math Optim. 2019, 80, 665-678. [CrossRef]

24. Van Hieu, D.; Anh, P.K.; Muu, L.D. Modified forward-backward splitting method for variational inclusions. 4OR-Q J. Oper. Res. 2020. [CrossRef]

25. Abbas, B.; Attouch, H.; Svaiter, B.F. Newton-Like Dynamics and Forward-Backward Methods for Structured Monotone Inclusions in Hilbert Spaces. J. Optim. Theory Appl. 2014, 161, 331-360. [CrossRef]

26. Attouch, H.; Cabot, A. Convergence of a Relaxed Inertial Forward-Backward Algorithm for Structured Monotone Inclusions. Appl. Math. Optim. 2019. [CrossRef]

27. Boţ, R.I.; Sedlmayer, M.; Vuong, P.T. A relaxed inertial Forward-Backward-Forward algorithm for solving monotone inclusions with application to GANs. arXiv 2020, arXiv:2003.07886.

28. Maingé, P.-E. Convergence theorems for inertial KM-type algorithms. J. Comput. Appl. Math. 2008, 219, $223-236$. [CrossRef]

29. Opial, Z. Weak convergence of the sequence of successive approximations for nonexpansive mappings. Bull. Am. Math. Soc. 1967, 73, 591-597. [CrossRef]

30. Xu, H.K. Iterative algorithms for nonlinear operators. J. London. Math. Soc. 2002, 66, 240-256. [CrossRef]

31. Barbu, V. Nonlinear Semigroups and Differential Equations in Banach Spaces; Editura Academiei RS Romania: Bucharest, Romania, 1976.

32. Cevher, V.; Vũ, B.C. A reflected Forward-Backward splitting method for monotone inclusions involving Lipschitzian operators. Set-Valued Var. Anal. 2020. [CrossRef]

33. Rieger, J.; Tam, M.K. Backward-Forward-Reflected-Backward splitting for three operator monotone inclusions. Appl. Math. Comput. 2020, 381, 125248. [CrossRef] 